\title{
Generation 60plus im Focus der Wissenschaft
}

Mit der letzten Ausgabe von HeilberufeSCIENCE haben wir es mit dem Artikel zum Online-Banking von Senioren bis in die Tagespresse und auf die Websites für Senioren geschafft ein toller Erfolg. Zeigt er doch, welchen Bedarf Pflege und Gesellschaft an Informationen über die Generation 60plus haben. Vielleicht gelingt uns das ja wieder? Denn mit der Übersichtsarbeit von Meiners et al. „Erfolgreichreiches Marketing für ältere Konsumenten" - starten wir diese neue Ausgabe von HeilberufeSCIENCE. Dieser Beitrag bildet die inhaltliche Fortsetzung zur Übersichtsarbeit von Meiners et al. "Megatrend Alter" in der vorangehenden Ausgabe. Daran schließt sich eine Übersichtsarbeit von K. von Lindeman et al. an, welche die aktuelle Studienlage der vorhandenen internationalen Studien zum Gesundheitsverhalten von Auszubildenden in Krankenpflegeberufen darstellt. Die folgende Originalarbeit von Maier und Koller präsentiert die Ergebnisse einer empirischen Untersuchung zum Bedarf an institutionalisierter psychologischer Beratung für das Personal in geriatrischen Pflegeeinrichtungen.

In Ordenskrankenhäusern arbeiten neben kirchlichen auch zivile Mitarbeiterlnnen, nicht zuletzt, weil die Ordensgemeinschaften seit Jahrzehnten stark rückläufige Eintrittszahlen verzeichnen. Grund genug für Stefinger et al., die Werteordnung dort und deren Unterschiede einmal unter die Lupe zu nehmen, um daraus Hinweise zur Reduktion von Konfliktpotenzialen abzuleiten.
Heckel et al. gehen in der folgenden Übersichtsarbeit der Frage nach, welche Bedeutung Geruch und Geschmack im Lebensverlauf älterer Menschen haben. Sie weisen darauf hin, dass die subjektive Bedeutung von Geruch und Geschmack im Lebensverlauf ein in der Gerontologie bisher kaum bearbeitetes Thema sei.

Den Abschluss bildet auch in dieser Ausgabe eine wissenschaftliche Kurzmitteilung. Ullrich et al. analysieren darin die Zusammenhänge zwischen dem Personalbestand und den bei Begehungen durch die Heimaufsicht festgestellten Defiziten in Altenpflegeeinrichtungen.

Auch diesmal möchte ich Sie an dieser Stelle wieder einladen, die drei Übersichtsartikel, zwei Originalarbeiten und den wissenschaftlichen Kurzbeitrag dieser Ausgabe von HeilberufeSCIENCE kritisch zu lesen. Vielleicht bieten Ihnen diese Artikel interessante Anregungen für Ihre eigene Tätigkeit oder eigenen Projekte?

Ich wünsche Ihnen noch eine schöne Sommerzeit und würde mich freuen, wenn ich Sie in der nächsten Ausgabe im November 2011 wieder an dieser Stelle begrüßen darf.

Ihr

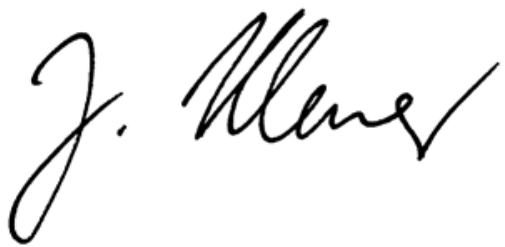

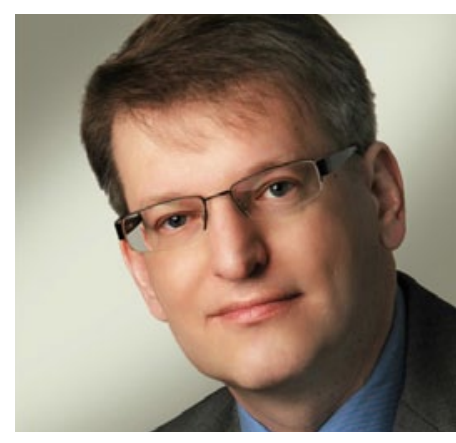

Prof. Dr. med. habil. Jörg Klewer, Zwickau

Schriftleitung

heilberufescience@springer.com

HeilberufeSCIENCE 2011; 2 (3): 71

DOI 10.1007/s16024-011-0340-0 